\title{
Advances in Studies and Applications of Centroidal Voronoi Tessellations
}

\author{
Qiang Du ${ }^{1, *}$, Max Gunzburger ${ }^{2}$ and Lili $\mathrm{Ju}^{3}$ \\ ${ }^{1}$ Department of Mathematics, Pennsylvania State University, University Park, PA \\ 16802, USA. \\ ${ }^{2}$ Department of Scientific Computing, Florida State University, Tallahassee, FL \\ 32306, USA. \\ ${ }^{3}$ Department of Mathematics, University of South Carolina, Columbia, SC 29208, \\ USA
}

\begin{abstract}
Centroidal Voronoi tessellations (CVTs) have become a useful tool in many applications ranging from geometric modeling, image and data analysis, and numerical partial differential equations, to problems in physics, astrophysics, chemistry, and biology. In this paper, we briefly review the CVT concept and a few of its generalizations and well-known properties. We then present an overview of recent advances in both mathematical and computational studies and in practical applications of CVTs. Whenever possible, we point out some outstanding issues that still need investigating.
\end{abstract}

AMS subject classifications: 5202, 52B55, 62H30, 6502, 65D30, 65U05, 65Y25, 68U05, 68U10

Key words: Voronoi tessellations, centroids, clustering, mesh generation and optimization, image processing, model reduction, point sampling.

\section{Introduction}

A comprehensive study of centroidal Voronoi tessellations (CVTs) was provided in the 1999 review article [31]. While the CVT concept initially was phrased as a model and method for optimal point distributions and spacial tessellations of regions/volumes in $\mathbb{R}^{d}$ or within sets of discrete data, the generality and universality of CVTs have also made it widely applicable in many fields of science and engineering. In the past decade, CVTs and CVT-based methodologies have attracted much attentions in the community. Not only has significant progress been made in the theoretical study of the CVTs, but there also has been tremendous growth in the scientific and technological applications of CVTs. The purpose of this paper is to reflect upon past progress and to point out some interesting issues that still need to be resolved.

Given the vast literature published on the subject, it is impossible to give a complete survey; instead, we focus on works with which we are most familiar to offer a brief and

${ }^{*}$ Corresponding author. Email addresses: qdu@math.psu.edu, gunzburg@fsu.edu, ju@math.sc. edu 
limited overview of the recent advances in mathematical and computational studies and in practical applications of CVTs. Needless to say, the subject of CVTs is still growing rapidly, so we also point out some outstanding issues and future research topics that remain to be studied.

The paper is organized as follows, in the remainder of this section, we go over the CVT concept and a few of its basic properties. Then, in Section 2, we discuss several generalizations of the basic CVT concept and, in Section 3, we discuss recent progress in the development of improved, i.e., more efficient algorithms, for constructing CVTs. In Section 4, we discuss a very few of the many and ever growing applications to which CVTs have been put to effective use. Brief concluding remarks are given in Section 5.

\subsection{The CVT concept in $\mathbb{R}^{d}$}

We first recall the definition of CVTs in Euclidean space. We begin with a given open bounded domain $\Omega \in \mathbb{R}^{d}$ and a set of distinct points $\left\{\mathbf{x}_{i}\right\}_{i=1}^{n} \subset \Omega$. For each point $\mathbf{x}_{i}$, $i=1, \ldots, n$, define the corresponding Voronoi region $V_{i}, i=1, \ldots, n$, by

$$
V_{i}=\left\{\mathbf{x} \in \Omega \mid\left\|\mathbf{x}-\mathbf{x}_{i}\right\|<\left\|\mathbf{x}-\mathbf{x}_{j}\right\| \quad \text { for } j=1, \cdots, n \text { and } j \neq i\right\},
$$

where $\|\cdot\|$ denotes the Euclidean distance (the $L^{2}$ metric) in $\mathbb{R}^{d}$. Clearly $V_{i} \cap V_{j}=\emptyset$ for $i \neq j$, and $\cup_{i=1}^{n} \bar{V}_{i}=\bar{\Omega}$ so that $\left\{V_{i}\right\}_{i=1}^{n}$ is a tessellation of $\Omega$. We refer to $\left\{V_{i}\right\}_{i=1}^{n}$ as the Voronoi tessellation (VT) of $\Omega$ [96] associated with the point set $\left\{\mathbf{x}_{i}\right\}_{i=1}^{n}$. A point $\mathbf{x}_{i}$ is called a generator; a subdomain $V_{i} \subset \Omega$ is referred to as the Voronoi region (or Voronoi diagram) corresponding to the generator $\mathbf{x}_{i}$. It is well-known that the dual tessellation (in a graph-theoretical sense) to a Voronoi tessellation of $\Omega$ is the Delaunay triangulation (DT).

Given a density function $\rho(\mathbf{x}) \geq 0$ defined on $\Omega$, for any region $V \subset \Omega$, the standard mass center (or centroid) $\mathbf{x}^{*}$ of $V$ is given by

$$
\mathbf{x}^{*}=\frac{\int_{V} \mathbf{x} \rho(\mathbf{x}) \mathrm{d} \mathbf{x}}{\int_{V} \rho(\mathbf{x}) \mathrm{d} \mathbf{x}} .
$$

Then a special family of Voronoi tessellations are defined as follows.

Definition 1. [31] We refer to a Voronoi tessellation $\left\{\left(\mathbf{x}_{i}, V_{i}\right)\right\}_{i=1}^{n}$ of $\Omega$ as a centroidal Voronoi tessellation (CVT) if and only if the points $\left\{\mathbf{x}_{i}\right\}_{i=1}^{n}$ which serve as the generators of the associated Voronoi regions $\left\{V_{i}\right\}_{i=1}^{n}$ are also the centroids of those regions, i.e., if and only if we have that $\mathbf{x}_{i}=\mathbf{x}_{i}^{*}$ for $i=1, \ldots, n$. The corresponding dual triangulation is called a centroidal Voronoi Delaunay triangulation (CVDT).

A generic Voronoi tessellation does not satisfy the CVT property in general; see Figure 1 for an illustration. On the other hand, given a density $\rho$ and a number of generators $n$, the CVT of a domain always exists though it may not be unique. 

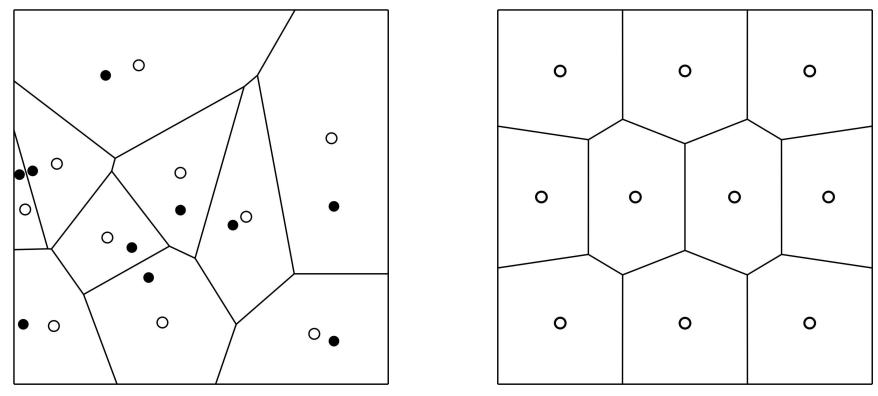

Figure 1: [31] A Voronoi tessellation of the unit square with 10 generators (the dots) randomly selected (the circles denote the centroids which do not coincide with the generators) and a 10-point centroidal Voronoi tessellation of the square for a uniform density.

\subsection{Basic properties of CVT}

CVTs possess an optimization property that can be used as a basis for their various extensions. Given any set of points $\widetilde{\mathbf{X}}=\left\{\widetilde{\mathbf{x}}_{i}\right\}_{i=1}^{n}$ in $\Omega$ and any tessellation $\widetilde{\mathbf{V}}=\left\{\widetilde{V}_{i}\right\}_{i=1}^{n}$ of $\Omega$, define a clustering energy by

$$
\mathbb{K}(\widetilde{\mathbf{X}}, \widetilde{\mathbf{V}})=\sum_{i=1}^{n} \int_{\widetilde{V}_{i}} \rho(\mathbf{x})\left\|\mathbf{x}-\widetilde{\mathbf{x}}_{i}\right\|^{2} \mathrm{~d} \mathbf{x} .
$$

Then it can be shown that $\mathbb{K}$ is minimized only if $\left\{\left(\widetilde{\mathbf{x}}_{i}, \widetilde{V}_{i}\right)\right\}_{i=1}^{n}$ forms a CVT of $\Omega$. We remark that if $\left\{\left(\widetilde{\mathbf{x}}_{i}, \widetilde{V}_{i}\right)\right\}_{i=1}^{n}$ forms a CVT it does not necessarily minimize $\mathbb{K}$; e.g., they may define a saddle point [31]. The energy functional $\mathbb{K}$ is often naturally associated with quantities such as quantization error, variance and cost in many applications.

CVTs can be constructed either using probabilistic methods typified by MacQueen's random algorithm [86] or deterministic methods typified by Lloyd's method [82]. Asymptotically as the number of generators gets larger and larger, Gersho's Conjecture [55] states that the optimal CVT (in the sense of minimizing the energy) forms a regular tessellation consisting of the replication of a single polytope whose shape depends only on the spatial dimension. The regular hexagon provides a confirmation of the conjecture in two dimensions for the case of CVTs associated with a constant density [91]. For the three-dimensional case, it was proved that among all lattice-based CVTs, the CVT corresponding to the body-centered cubic lattice $\mathbf{D}_{3}^{*}$ (its Voronoi regions are the space-filling truncated octahedra) is the optimal one [18]. For more general, non-lattice cases with nonconstant densities, the question remains open, although extensive numerical simulations given in [47] demonstrated that the truncated octahedra is the likely candidate.

\section{Generalizations of Centroidal Voronoi Tessellations}

The CVT concept can be generalized to very broad settings that range from abstract spaces and distance metrics to discrete point sets [31]. Some of these generalizations are reviewed below. 


\subsection{CVTs of surfaces}

To incorporate constraints on the generators into CVTs, the notion of constrained CVTs was first considered in [32] for the mesh generation setting using a mixed variational formulation. A more systematic extension of the CVT concept to surfaces (or manifolds) was considered in [36] under the Euclidean metric.

Suppose that $\mathbf{S}$ is a compact and continuous hyper-surface in $\mathbb{R}^{d+1}$, then for any region $V \subset \Omega$, we call $\mathbf{x}^{c}$ a constrained mass center of $V$ if

$$
\mathbf{x}^{c}=\arg \min _{\mathbf{x} \in V} \int_{V} \rho(\mathbf{y})\|\mathbf{y}-\mathbf{x}\|^{2} \mathrm{~d} \mathbf{y} .
$$

Existence of minimizers of the problem (2.1) can be easily obtained using the continuity and compactness of the objective function; however, solutions may not be unique depending on the curvature of $V$. It is worth noting that if $\mathbf{S}$ is a flat surface, then $\mathbf{x}^{c}$ coincides with $\mathbf{x}^{*}$, the standard mass center of $V$.

Definition 2. [36] We refer to a Voronoi tessellation $\left\{\left(\mathbf{x}_{i}, V_{i}\right)\right\}_{i=1}^{n}$ of a surface $\mathbf{S}$ as a constrained centroidal Voronoi tessellation (CCVT) if and only if we have that $\mathbf{x}_{i}=\mathbf{x}_{i}^{c}$ for $i=1, \ldots, n$. The corresponding dual triangulation is called a constrained centroidal Voronoi Delaunay triangulation (CCVDT).

In particular, when $\Omega$ is the surface of a sphere, we call $\left\{\left(\mathbf{x}_{i}, V_{i}\right)\right\}_{i=1}^{n}$ a spherical centroidal Voronoi tessellation (SCVT). Figure 2 presents some sample CCVTs on the a sphere and torus where, in the case of a sphere, the construction of spherical Voronoi diagrams was efficiently implemented based on Lloyd's method with the help of the software package "STRIPACK" [105].
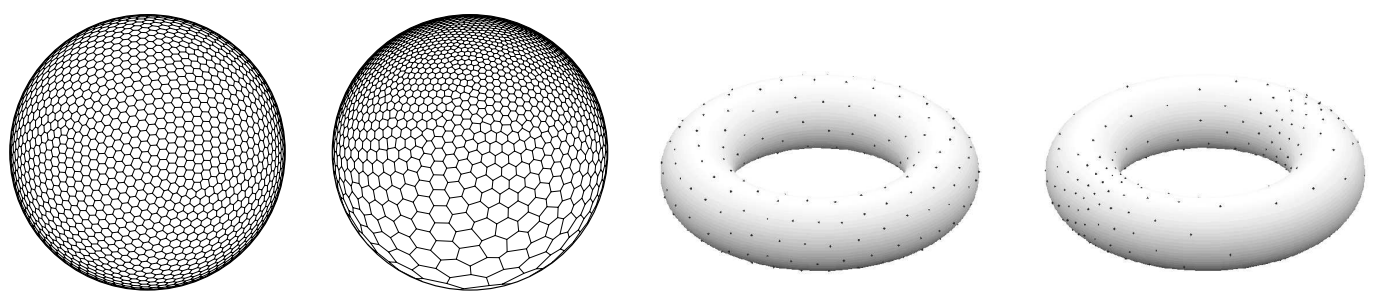

Figure 2: [36] CCVT point placement on surfaces. From left to right: centroidal Voronoi cells for 2562 points on the sphere distributed uniformly and non-uniformly, 256 points on a torus surface distributed uniformly and non-uniformly, respectively.

\subsection{CVTs under other metrics}

CVTs may be defined for metrics other than the Euclidean distance. For example, in Figure 3, we give examples of CVTs of a square under the $L^{1}(\Omega), L^{20}(\Omega)$, and a triangle metrics. Probabilistic algorithms for the construction of CVTs are especially useful for such 
metrics because such methods rely only on determining the distance between points and not on the explicit construction of Voronoi diagrams or of centers of mass; see Section 3.2.
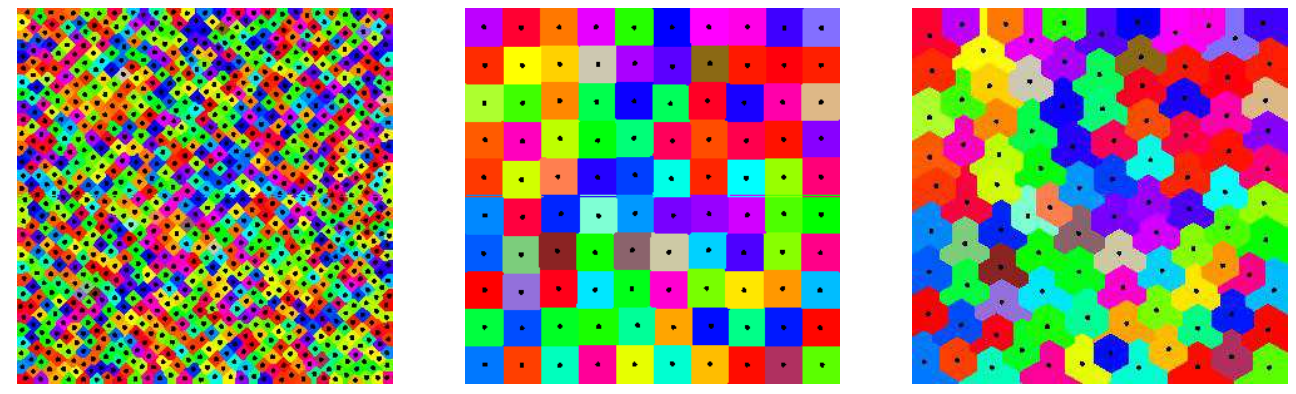

Figure 3: [Courtesy of Hoa Nguyen] From left to right: CVTs with a uniform density under the $L^{1}$ metric, the $L^{20}$ metric (approximating the $L^{\infty}$ metric), and the triangle metric.

\subsection{CVTs of line segments}

Generalized Voronoi tessellations for which the generators are line segments or areas are defined in [96]. In [65], line segment generators were used for stippling applications. Generalization of the CVT concept to line segments and graphs via a variational characterization was proposed in [84]. Such a generalization is likely to have several applications such as skeleton fitting and segmentation and vector field visualization [51]. Let $\mathbf{E}=\left\{e_{i}=\left[\mathbf{x}_{i_{1}}, \mathbf{x}_{i_{2}}\right]\right\}_{i=1}^{n}$ denote a set of line segments having end points in $\mathbf{X} \subset \Omega$. The Voronoi region $V_{e_{i}}$ in $\Omega$ associated with the segment $e_{i}=\left[\mathbf{x}_{i_{1}}, \mathbf{x}_{i_{2}}\right]$ is defined by

$$
V_{e_{i}}=\left\{\mathbf{x} \in \Omega \mid d\left(\mathbf{x}, e_{i}\right)<d\left(\mathbf{x}, e_{j}\right) \text { for } j=1, \cdots, n \text { and } j \neq i\right\}
$$

where $d\left(\mathbf{x}, e_{i}\right)$ denotes the Euclidean distance from the point $\mathbf{x}$ to the segment $e_{i}$. The clustering energy with respect to a line segment $e_{i}=\left[\mathbf{x}_{i_{1}}, \mathbf{x}_{i_{2}}\right]$ can be defined as $F_{s}\left(e_{i}\right)=$ $\int_{V_{e_{i}}} d^{2}\left(\mathbf{x}, e_{i}\right) \mathrm{d} \mathbf{x}$. Then the Voronoi tessellation $\mathbf{V}=\left\{V_{e_{i}}\right\}_{i=1}^{n}$ of $\Omega$ associated with the line segments (E, $\mathbf{X})$ is called a CVT [84] if $\mathbf{X}$ is a minimizer of the following energy

$$
\mathbb{F}_{s}(\mathbf{X})=\sum_{e_{i} \in \mathbf{E}} F_{s}\left(e_{i}\right)
$$

We specially note that the connectivity information $\mathbf{E}$ is pre-given and fixed in the minimization. In addition, only the uniform density is considered in this case. Some efficient approximation algorithms for computing Voronoi regions of line segments and the construction of CVTs of line segments were proposed in [84].

Examples of CVTs for line segment and mixed line segment/point generators are presented in Figure 4. 

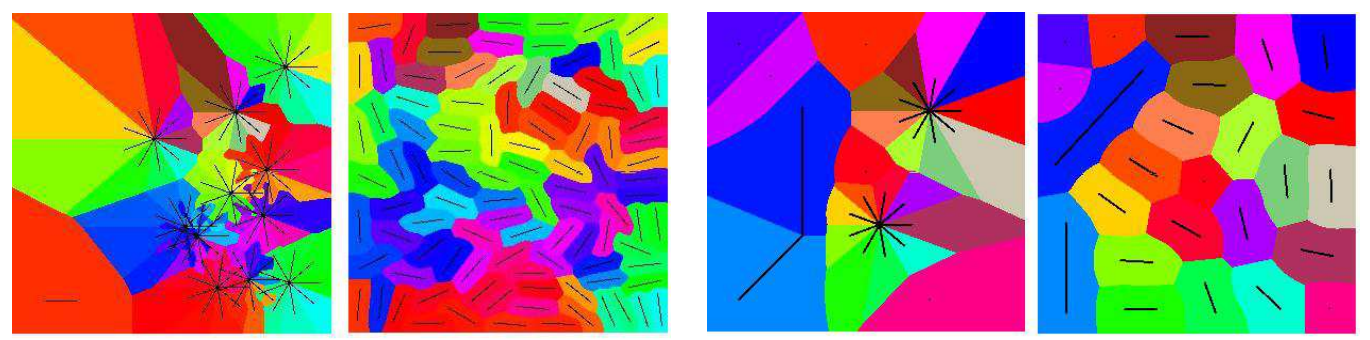

Figure 4: [Courtesy of Hoa Nguyen] Left pair: VT of initial distribution of line segments and final CVT configuration; right pair: VT of initial distritution of points and line segments and final CVT configuration.

\subsection{Anisotropic CVTs}

To account for more general Riemannian metric, the concept of anisotropic centroidal Voronoi tessellation was proposed in [46]; see also [93]. Let $\mathbf{p} \in \Omega$ be a point, then a metric tensor at $P$ refers to a symmetric positive matrix (tensor) $M(\mathbf{p})$ which induces a Riemannian structure on $\Omega$, denoted by $(\Omega, M(\mathrm{p}))$. We may define the following directional distance from $\mathbf{q}$ to $\mathbf{p}$ as

$$
d_{\mathbf{p}}(\mathbf{p}, \mathbf{q})=\sqrt{\overrightarrow{\mathbf{p q}}^{T} M(\mathbf{p}) \overrightarrow{\mathbf{p q}}}
$$

This directional distance between $\mathbf{p}$ and $\mathbf{q}$ can be interpreted as a Riemannian distance between the two points, with the metric being a constant metric given by $M(\mathbf{p})$. In contrast to the usual distance definitions, this directional distance is not symmetric. The anisotropic Voronoi region (AVR) $V_{j}$ corresponding to the point $\mathbf{x}_{i}$ is then defined by

$$
V_{i}=\left\{\mathbf{x} \in \Omega \mid d_{\mathbf{x}}\left(\mathbf{x}, \mathbf{x}_{i}\right)<d_{\mathbf{x}}\left(\mathbf{x}, \mathbf{x}_{j}\right), \quad \text { for } j=1, \cdots, n \text { and } j \neq i\right\} .
$$

Then $\left\{V_{i}\right\}_{i=1}^{K}$ forms an anisotropic Voronoi tessellation (AVT) of $\Omega$. Note that AVRs may not be a polygonal or convex or singly-connected. An anisotropic energy on a region $V$ can be defined as $F_{a}(\mathbf{x})=\int_{V} d_{\mathbf{y}}^{2}(\mathbf{y}, \mathbf{x})$ dy, and consequently the anisotropic mass center $\mathbf{x}^{a}$ of $V$ is given by

$$
\mathbf{x}^{a}=\left[\int_{V} M(\mathbf{x}) \mathrm{d} \mathbf{x}\right]^{-1}\left[\int_{V}(M(\mathbf{x}), \mathbf{x}) \mathrm{d} \mathbf{x}\right]
$$

which minimizes $F_{a}(\mathbf{x})$. We note that, in contrast to standard CVTs, the density of AVTs can be mixed with the Riemannian metric $M$.

Definition 3. [46] An anisotropic Voronoi tessellation $\left\{\left(\mathbf{x}_{i}, V_{i}\right)\right\}_{i=1}^{n}$ of a domain $\Omega$ under the metric tensor $M$ and the directional distance function $d_{\mathbf{p}}$ is called an anisotropic centroidal Voronoi tessellation (ACVT) if $\mathbf{x}_{i}=\mathbf{x}_{i}^{a}, i=1, \cdots, n$. The corresponding dual triangulation is referred to as the anisotropic centroidal Voronoi Delaunay triangulation (ACVDT).

A corresponding minimization property can be derived for ACVTs under the following energy:

$$
\mathbb{F}_{a}(\tilde{\mathbf{X}}, \widetilde{V})=\sum_{i=1}^{n} \int_{V_{i}} d_{\mathbf{x}}^{2}\left(\mathbf{x}, \mathbf{x}_{i}\right) \mathrm{d} \mathbf{x} .
$$


Numerical examples were provided in [46] together with an application to surface anisotropic meshing. Figure 2 presents some sample ACVTs produced by the algorithms provided there. See Section 4.3 for additional examples .
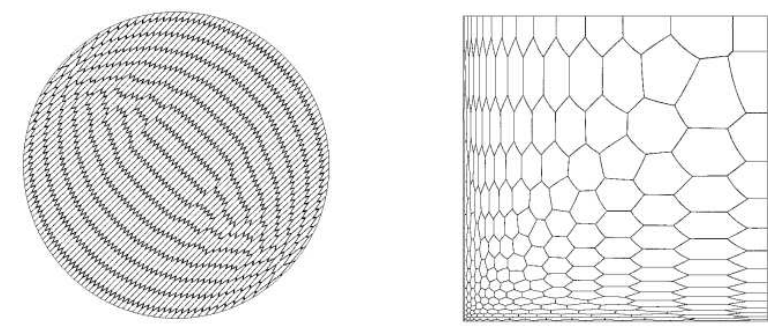

Figure 5: [46] ACVTs of a circle (left) and a square (right) with some metric tensors.

The idea of a one-sided distance was used again in the work of [51] for CVT-based vector field simplification and visualization.

Interestingly, the one-sided distance can also be used to define a different notion of anisotropic Voronoi regions via

$$
V_{i}=\left\{\mathbf{x} \in \Omega \mid d_{\mathbf{x}_{i}}\left(\mathbf{x}_{i}, \mathbf{x}\right)<d_{\mathbf{x}_{j}}\left(\mathbf{x}_{j}, \mathbf{x}\right), \text { for } j=1, \cdots, n \text { and } j \neq i\right\} .
$$

The associated anisotropic Voronoi tessellations have been discussed in [76]. One can naturally define the corresponding anisotropic centroidal Voronoi tessellations. Note that this includes the special cases of weighted centroidal Voronoi tessellations, that is, $\left\{d_{\mathbf{x}_{i}}\left(\mathbf{x}_{i}, \mathbf{x}\right)=\right.$ $\left.m_{i}\left\|\mathbf{x}-\mathbf{x}_{i}\right\|\right\}$ for some constants $\left\{m_{i}\right\}$, as well as ones that use general covariance matrices $\left\{M_{i}\right\}$ to $\left\{d_{\mathbf{x}_{i}}\left(\mathbf{x}_{i}, \mathbf{x}\right)=\left(\mathbf{x}-\mathbf{x}_{i}\right)^{T} M_{i}\left(\mathbf{x}-\mathbf{x}_{i}\right)\right\}$. The latter has been used in [50] to connect the generalized Lloyd's iterations to the mixture clustering models for data mining.

More generally, the two types of anistropic Voronoi tessellations can all be unified through the so call Bregman Voronoi tessellations and one can thus discuss a more general centroidal Bregaman Voronoi tessellations, see [92] for more details.

\section{Algorithmic Advances}

Along with many applications whose number and diversity are still growing, significant advances have been made in the design of efficient and robust algorithms for computing CVTs $[27,28,69,74,81]$ and in their convergence analysis $[28,29,52,53]$.

\subsection{Convergence of Lloyd's method}

Due to its effectiveness and simplicity, much attention has been focused on Lloyd's method.

Algorithm 1. (Lloyd's Method) Given a domain $\Omega$, a density function $\rho$ defined on $\Omega$, and a positive integer $n$. 
0. Select an initial set of $n$ points $\left\{\mathbf{x}_{i}\right\}_{i=1}^{n}$ on $\Omega$;

1. Construct the Voronoi regions $\left\{V_{i}\right\}_{i=1}^{n}$ of $\Omega$ associated with $\left\{\mathbf{x}_{i}\right\}_{i=1}^{n}$;

2. Determine the centroids (or constrained centroids) of the Voronoi regions $\left\{V_{i}\right\}_{i=1}^{n}$; these centroids form the new set of points $\left\{\mathbf{x}_{i}\right\}_{i=1}^{n}$;

3. If the new points meet some convergence criterion, return $\left\{\left(\mathbf{x}_{i}, V_{i}\right)\right\}_{i=1}^{n}$ and terminate; otherwise, go to Step 1.

The Lloyd algorithm is also called the $k$-means algorithm in the clustering and quantization fields $[58,74]$. It is worth noting that the energy $\mathbb{K}$ associated with the Voronoi tessellation decreases monotonically during Lloyd iterations until a CVT is reached [31].

Let the Lloyd map be the mapping from a set of distinct generators $\mathbf{X}=\left(\mathbf{x}_{1}, \cdots, \mathbf{x}_{n}\right) \in$ $\Omega^{n} \subset \mathbb{R}^{n \times d}$ to the corresponding centroids, defined by $\mathbf{T}=\left(\mathbf{T}_{1}, \ldots, \mathbf{T}_{n}\right)^{T}$ such that

$$
\mathbf{T}_{i}(\mathbf{X})=\frac{\int_{V_{i}(\mathbf{X})} \mathbf{x} \rho(\mathbf{x}) d \mathbf{x}}{\int_{V_{i}(\mathbf{X})} \rho(\mathbf{x}) \mathrm{d} \mathbf{x}}, \quad i=1, \cdots n,
$$

where $V_{i}(\mathbf{X})$ denotes the Voronoi region corresponding to $\mathbf{x}_{i}$ of the Voronoi tessellation having $\mathbf{X}$ as its generators. Then the Lloyd's algorithm may be viewed as a fixed point iteration of the Lloyd map: $\mathbf{X}^{(k+1)}=\mathbf{T}\left(\mathbf{X}^{(k)}\right)$. Let us define a new energy function $\mathbb{H}$ by

$$
\mathbb{H}(\mathbf{X}, \mathbf{Y}):=\mathbb{K}(\mathbf{Y}, \mathbf{V}(\mathbf{X}))=\sum_{i=1}^{n} \int_{V_{i}(\mathbf{X})} \rho(\mathbf{x})\left\|\mathbf{x}-\mathbf{x}_{i}\right\|^{2} \mathrm{~d} \mathbf{x} .
$$

Then Lloyd's method is in fact an alternating variable algorithm for minimizing $\mathbb{H}$, i.e., one alternates between minimizing $\mathbb{H}(\mathbf{X}, \mathbf{Y})$ with respect to $\mathbf{X}$ and $\mathbf{Y}$ [27]. Since Lloyd's pioneering work, many studies have been made on the convergence properties of the Lloyd iteration. Most notably, in [54] and later in [73], it was shown that the Lloyd iteration is a local contraction (thus locally convergent) in one dimension provided the density function is logarithmically concave, i.e., $(\log \rho)^{\prime \prime}<0$. This result was extended to all continuous and positive densities in the one-dimensional setting of scalar quantization in [119]. In higher dimensions, convergence results are much more limited. In $[59,114]$, convergence of the energy function was shown by defining values of the Lloyd map on degenerate points.

On the other hand, in $[29,53]$, the authors applied the theory of descent methods to Lloyd's method without any extension to degenerate points by showing that points of degeneracy locally maximize the energy. This is necessary to ensure that limit points are in fact CVTs. It was proved in [29] that if the iterates in the Lloyd method stay in a compact set where the Lloyd map $\mathbf{T}$ is continuous, then the algorithm is weakly globally convergent, i.e., any limit point of $\left\{\mathbf{X}^{(k)}\right\}_{k=0}^{\infty}$ is a critical point of $\mathbb{G}$, where $\mathbb{G}(\mathbf{X})=\mathbb{H}(\mathbf{X}, \mathbf{X})$ is called the quantization energy of $\mathbf{X}$. The compactness of the iteration seems to be intuitively true, but it has not been rigorously justified in the literature. The difficulty lies in showing that during the Lloyd iteration the generators do not get arbitrarily close as the Lloyd map is 
not well defined at degenerate points where some of the generators may coincide [85]. In one dimension, for $\Omega=[a, b]$ and a smooth density function $\rho>0$, it was shown in [29] that

$$
\min _{i=1, \cdots, n} \operatorname{diam}\left(V_{i}\left(\mathbf{X}^{(k)}\right)\right)>\frac{b-a}{2 n 4^{2 n-1}\left(M_{c}\right)^{n}} \text { for } k>n,
$$

where $M_{c}=\max _{\mathbf{x} \in \Omega} \rho(\mathbf{x}) / \min _{\mathbf{x} \in \Omega} \rho(\mathbf{x})$ and consequently Lloyd's method is weakly globally convergent. For higher dimensional spaces, it was recently proved in [53] that any limit point of the Lloyd iteration in any dimension is non-degenerate provided that $\Omega$ is a convex and bounded set and $\rho$ belongs to $L^{1}(\Omega)$ and is positive almost everywhere. Practically, this ensures that any limit point of the algorithm does generate a CVT. Note that the regularity requirement on the density function in [53] is much relaxed.

\subsection{Probabilistic Lloyd algorithms and their parallel implementation}

Efficient and robust construction of CVTs are crucial to the success of utilizing the CVT concept in various applications. Among the advantages of the CVT methodology is that points may be easily distributed according to a prescribed density function and the algorithms which make up the methodology are amenable to parallelization. This was partly accomplished in [69] where some superior probabilistic CVT construction algorithms were introduced, implemented, and tested. These algorithms can be viewed as generalizations of both the simple MacQueen random and Lloyd deterministic algorithms, thus we call them probabilistic Lloyd algorithms. The key to the efficiency of the new methods is that many points are sampled and clustered before each averaging step is performed. It is very important to point out that these probabilistic algorithms do not require, at any stage, the construction of Voronoi diagrams nor the determination of the centroids of the Voronoi cells. Moreover, the algorithms are highly amenable to fully scalable parallelization, as was demonstrated in [69]. Figure 6 shows results of some experiments carried out for one of the probabilistic Lloyd algorithms in [69]. We see from the figure the nearly perfect speed up of the algorithm, independent of the density function $\rho$. The methods presented constitute a very efficient methodology for constructing CVTs and, as a result, in many applications they render the CVT concept superior to other approaches.

\subsection{Multi-level and Newton-type methods}

Like many iterative methods, the performance of Lloyd's method depends on the initial guess for the generators. Some good initialization processes can improve the speed and results of the Lloyd iteration [90]. Meanwhile, it was also known that the reduction of the errors in the Lloyd iteration eventually slows down as the iteration number increases $[28,31]$ because the errors become more smoothly distributed over the domain. To accelerate its local convergence, a nonlinear multilevel energy-based quantization algorithm was proposed and analyzed in [28] which combines the Lloyd iteration, a spatial decomposition and a multilevel successive subspace corrections. Each of the correction procedures involves solving a system of nonlinear equations. The multilevel algorithm 

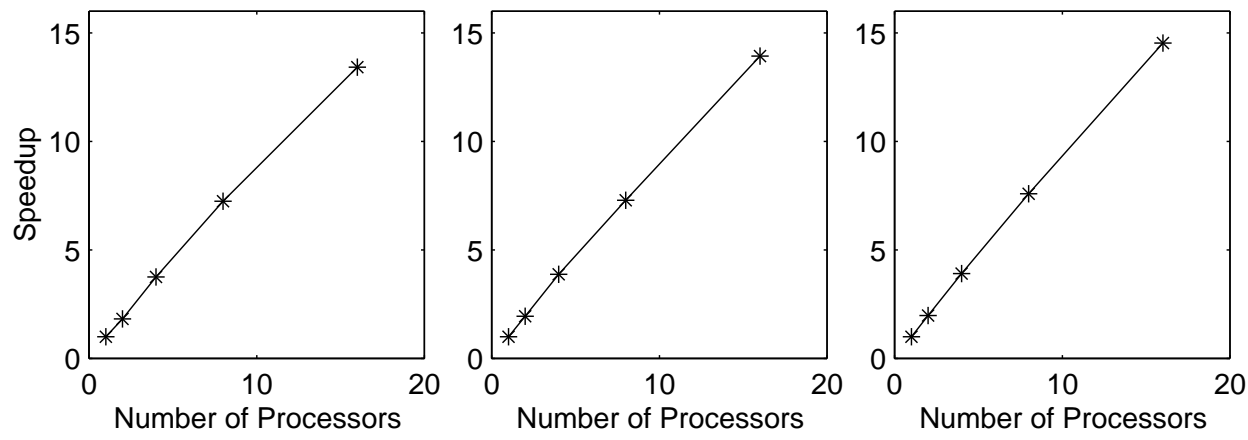

Figure 6: [69] Speed-up of a probabilistic Lloyd algorithm for three different density functions in one dimension. From left to right: $\rho(x)=1, \rho(x)=e^{-10 x^{2}}, \rho(x)=e^{-20 x^{2}}+0.05 \sin ^{2}(\pi x)$.

performs well in accelerating convergence near a local minimum but it does not change the linear convergence nature nor helps with the global energy minimization.

Given the linear convergence of Lloyd-like methods [29,31], there have been much effort directed at developing effective algorithms having higher convergence rates. In [27], a Lloyd-Newton algorithm was proposed to accelerate the construction of CVTs. The main idea is that one first runs the standard Lloyd algorithm until either the change in the energy $\mathbb{G}$ or the change in the displacements of the generators between two (or more) successive iterates is small, then one switches to a Newton iteration. The Newton algorithms are generally quadratically convergent but are also very sensitive to the initial guess. In practice, the Newton iteration in [27] takes the form

$$
\mathbf{X}^{(k+1)}=\mathbf{X}^{(k)}+\left(\mathrm{d} \mathbf{T}\left(\mathbf{X}^{(k)}\right)-\mathbf{I}\right)^{-1}\left(\mathbf{X}^{(k)}-\mathbf{T}\left(\mathbf{X}^{(k)}\right)\right) .
$$

Note that $\mathrm{d} \mathbf{T}$ denotes the Jacobian matrix of $\mathbf{T}$ (an $n d \times n d$ matrix) with its $((i-1) d+$ $m,(l-1) n+j)$-th entry given by $\partial \mathbf{T}_{i}^{m} / \partial \mathbf{x}_{j}^{l}$, where $\mathbf{T}_{i}^{m}$ denotes the $m$-th component of $\mathbf{T}_{i}$ and $\mathbf{x}_{j}^{l}$ the $l$-th component of $\mathbf{x}_{j}$. Major concerns about the Newton algorithm include the storage of $\mathrm{d} \mathbf{T}$ and the solution process for the resulting large-scale linear system when the number of generator $n$ is very large.

To avoid the computation and storage of the Jacobian matrix in the Newton iteration, it was suggested in [31] that Newton-like or quasi-Newton method can be a better alternative. This is achieved in a recent work of [81] based on the preconditioned limited memory $B F G S$ (L-BFGS) method [80]. In addition, the authors proved that $\mathbb{G}$ is $C^{2}$ (i.e., $\mathbf{T}$ is $C^{1}$ ) in any convex domain in the two or three dimension with a $C^{2}$ smooth density function; when the domain is non-convex, $\mathbb{G}$ is still $C^{2}$ in most cases except for some cases rarely encountered in practice where it becomes $C^{1}$. This provides the necessary justification for developing efficient Newton or quasi-Newton methods for accelerating CVT computations. Fast performance and super robustness of this method was shown by extensive experimental examples in various cases in two- and three-dimensional examples. 


\section{Progress in Applications of Centroidal Voronoi Tessellations}

CVT-based techniques have proven to be very useful in diverse applications, including but not limited to, image processing and analysis [20, 26, 38, 51, 57, 102, 121], vector quantization and data analysis [50,55, 56,58-60,78], resource optimization [31], optimal placement of sensors and actuators for control [24,31], cell biology and territorial behavior of animals [8], model reduction [11, 12,33,34,77], point sampling [13, 30, 97, 107, 115], meshless computing [21,35], mesh generation and optimization $[1,2,32,36,44-47,49$, $68,88,93,94,116,120]$, numerical partial differential equations [32, 37, 40-43, 66, 70$72,94,120]$, geophysical flows $[79,106,108]$, computer graphics $[5,17,19,39,61,65,81$, $81,81,110,112,116-118]$, mobile sensing networks [10,22, 24], logistics system design $[98,99]$, and phyllotaxis $[63,64]$. Obviously we cannot review all of these applications so we content ourselves with a representative sample.

\subsection{Mesh generation and optimization}

Mesh generation often forms a crucial part of the numerical solution procedure in many applications, especially numerical partial differential equations (PDEs) based applications. For unstructured mesh generation, some of the well-studied techniques include advancing front technique [83], Octree [113], Voronoi/Delaunay-based methods [7,16,25,111], and DistMesh [104]. When CVT/CVDT is applied to the numerical solution of PDEs, some modifications are needed to handle geometric constraints; for example, an obvious one is that some of the CVT generators (vertices of the corresponding CVDT) have to be constrained to lie on the boundary of the domain so that the boundary conditions of the problem can be enforced. For this purpose, [32] proposed the notion of constrained CVTs and discussed various approaches to deal with generators on the boundary, including projections from the interior to boundary or lifting from the boundary to the interior or a mixed variational formulation. Various modifications to the Lloyd's method were also presented. Extensions to three dimensions and optimizations in combination with vertex/face swappings were studied in $[1,44,45,48]$ to improve mesh quality; see Figure 7 for some sample CVDT meshes in three dimensions. A brief overview of the generation of three-dimensional constrained CVDT meshes can be found in [49].

In $[68,70]$, the concept of conforming CVT/CVDT was proposed with regard to these constraints which further elaborated the mixed energy minimization formulation given in [32]. Assume that the domain $\Omega$ is compact and $\partial \Omega$ is piecewise smooth with singular/corner points $P_{S}=\left\{\mathbf{x}_{i}\right\}_{i=1}^{k}$. Denote by $\operatorname{Proj}(\mathbf{x})$ the process that projects $\mathbf{x} \in \Omega$ to the point on the boundary closest to $\mathbf{x}$. Denote by $P_{I}$ the set of generators whose Voronoi regions are interior to $\Omega$ and by $P_{B}$ the set of generators whose Voronoi regions extend to the boundary.

Definition 4. [68] A Voronoi Tessellation $\left\{\left(\mathbf{x}_{i}, V_{i}\right)\right\}_{i=1}^{n}$ of $\Omega$ is called a conforming centroidal Voronoi tessellation (CfCVT) if the following properties are satisfied:

$$
\text { - } P_{S} \subset\left\{\mathbf{x}_{i}\right\}_{i=1}^{n} ; \quad \bullet \mathbf{x}_{i}=\mathbf{x}_{i}^{*} \text { if } \mathbf{x}_{i} \in P_{I} ; \quad \bullet \mathbf{x}_{i}=\operatorname{Proj}\left(\mathbf{x}_{i}^{*}\right) \text { if } \mathbf{x}_{i} \in P_{B}-P_{S} .
$$



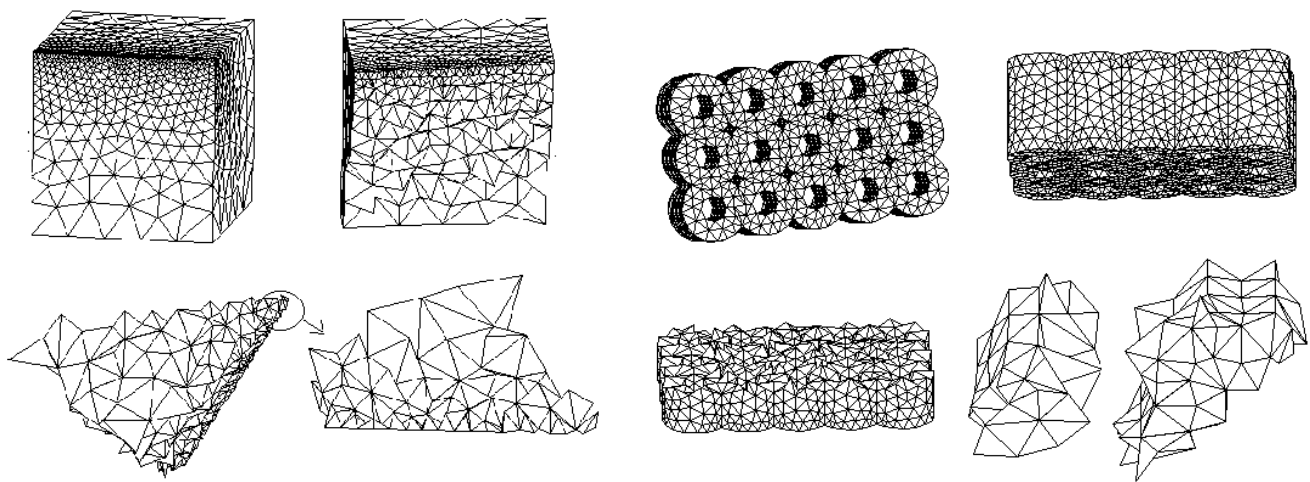

Figure 7: [44] Three-dimensioanl CVDT meshes of a cube (left pair) and a more complicated domain (right pair).

The dual triangulation is called a conforming centroidal Voronoi Delaunay triangulation (CfCVDT).

A special lifting process $[68,70]$ is added during the Lloyd iteration that allows the boundary generators to return to the interior domain together with the projection process so that the number of boundary generators can vary freely according to the density function. Thus the dependence of resulting CfCVDT meshes on the initial guess is greatly weakened. Figure 8 presents some sample CfCVDT meshes in two dimensions. Quality comparisons of the CfCVDT meshing scheme with some other triangular mesh generators are done in [93] and shows the superiority of this method in most cases.
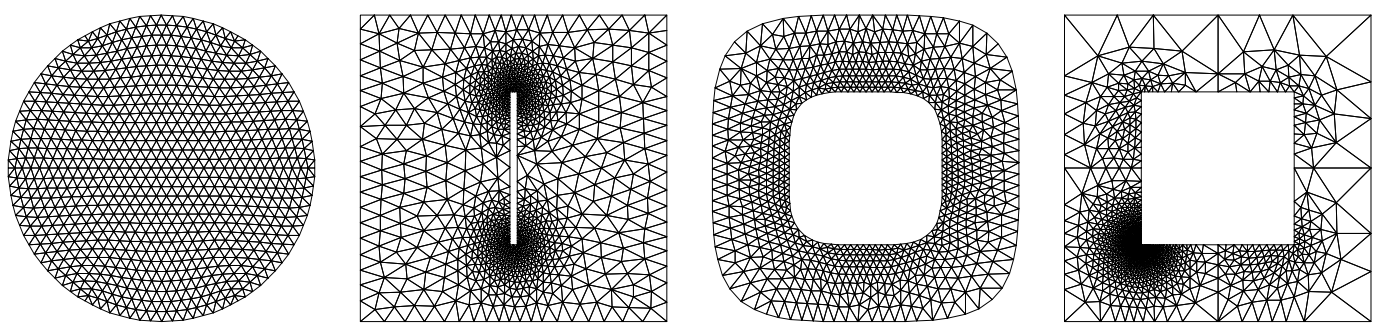

Figure 8: [68] Two-dimensional CfCVDT meshes for various domains and density functions.

In addition, CVT/CCVT based techniques have been used for mesh generation for numerical PDEs and surface re-meshing and optimization in the field of computer graphics $[5,17,90,102,116-118,120]$.

\subsection{CVT-based finite volume and finite element methods}

Finite volume methods in the plane are widely used in engineering applications. A Voronoi-based finite volume scheme for the discretization of PDEs on the sphere was developed based on spherical Voronoi tessellations [37]. For a model convection-diffusion 
problem, the Voronoi-based finite volume scheme is shown to produce first-order accurate approximations with respect to a mesh-dependent discrete first-derivative norm, and in particular, the $L^{2}$ error of the approximation is of quadratic order when the underlying Voronoi mesh is given by a spherical centroidal Voronoi tessellation (SCVT) [43]. Moreover, superconvergence of the approximate solutions was also numerically demonstrated. In [42] another novel finite volume discretization scheme was developed for the approximations of a reduced Ginzburg-Landau phenomenological model for superconducting hollow spheres based on SCVT [42] and used to study the behavior of the quantized vortices [41] on a spherical geometry. The scheme utilizes the properties of the SCVT and the discrete gauge invariance automatically is satisfied to accurately compute the various solution branches, vortex nucleation patterns and vortex dynamics on the sphere.

Stimulated by the above results, nonuniform SCVT/SCVDT grids were recently studied and applied for ocean and ice modeling in [108]; see Figure 9 (left) for SCVDT grids for the North Atlantic where the fourth power of the kinetic energy was used as a proxy to define the density function used in the SCVT/SCVDT construction. It suffices to note that SCVT/SCVDT grids take very good advantage of whatever proxy one chooses. The density function as well as the need to resolve boundaries motivate the variations in the grid size. Numerical examples reported in [108] based on the nonlinear shallow-water equations spanning the entire surface of the sphere elucidate both the potential benefits of this variable-resolution method and the challenges ahead; see Figure 9 (right) that shows the parallel simulation result of a standard shallow-water test case (test case 5 of [122] for which a flow in geostrophic balance is confronted with a large-scale orographic feature at the start of the simulation) on a nonuniform SCVT mesh using Voronoi-based finite volume discretization. This example clearly illustrates both the potential benefits of this SCVT based multi-resolution method and the challenges ahead.
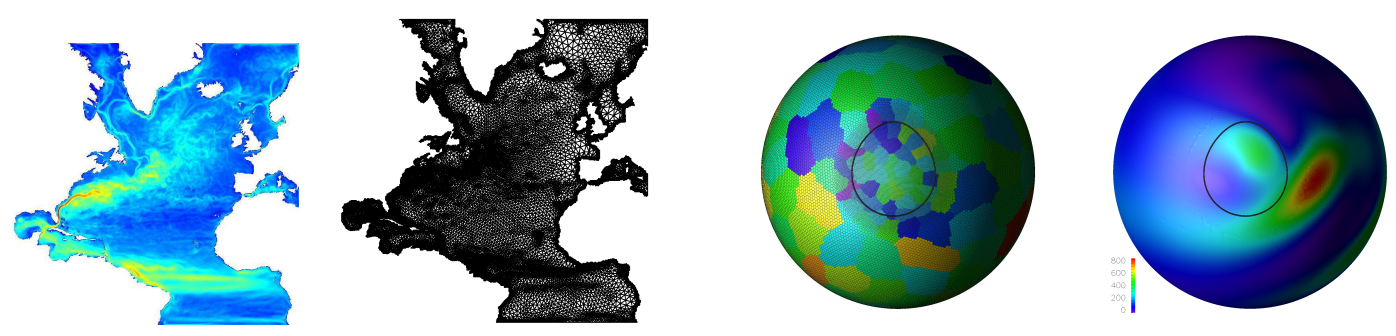

Figure 9: [108] Left pair: the original digital image of the kinetic energy in the North Atlantic and the corresponding SCVDT mesh; right pair: A nonuniform SCVT mesh (decomposed for parallel simulation) and the kinetic energy field at day 10 of the simulation based on this mesh.

Given the superior quality of the CVT/CVDT mesh, it is not a coincidence that the resulting finite volume methods exhibit superconvergent properties as observed in [43]. Similar studies on the superconvergence of finite element solutions based on the CVT/CVDT meshes were presented in [66]. 


\subsection{CVT-based adaptive algorithms for numerical PDEs}

Adaptive finite element and finite volume methods for the numerical solution of PDEs have been well developed in the past decades $[4,89]$. The essential ingredients are a posteriori error estimates and mesh refinement (or possible coarsening) schemes. The key step of adaptive methods is to choose reliable and efficient error estimators to refine the old mesh such that the errors of the approximate solutions on the new mesh can be distributed as uniformly as possible and the numerical solution converges to the analytic solutions with simple complexity when the mesh size goes to zero.

Adaptive finite element methods based on the CVT/CVDT meshes have been proposed in [32]. An interesting asymptotic relation between the density function and the local mesh size of a CVT is given by

$$
h_{i} / h_{j} \approx\left(\rho\left(\mathbf{x}_{j}\right) / \rho\left(\mathbf{x}_{i}\right)\right)^{1 /(d+2)},
$$

where $h_{i}$ denotes the diameter of the Voronoi region $V_{i}$ corresponding to $\mathbf{x}_{i}$. We remark that (4.1) is still a conjecture in spaces with $d \geq 2$ but has been numerically verified by extensive experiments and is widely assumed in practical applications such as vector quantization and image processing [58]. The main idea of CVT/CVDT based adaptive algorithms is to first refine the old mesh and then optimize the mesh using the CVT/CVDT algorithms according to either the solution norms given in [32] or some other local a posteriori error estimators $[67,70]$. One can explicitly determine a density function $\rho$ based on some a posteriori error estimator and the CVT mesh size relation (4.1) to generate the new mesh so that the errors of the new approximate solution will be equally distributed over the element in an optimal way $[32,67,70]$. In particular, an efficient nearest neighbor search algorithm proposed in [3] was used in [70] for the fast construction of the piecewise-smooth density function $\rho$ over the triangulated domain. It is worth noting that the density function $\rho$ and the resulting meshes could be different if different error estimators are used for the same problem, e.g., $H^{1}$-type vs. $L^{2}$-type estimators. Another advantage of these methods is that the resulting CVT/CVDT mesh always has good quality while most mesh adaptivity methods often degenerate the mesh quality to some extent as one refines. The study of CVT-based adaptive meshing methods has been done for second-order elliptic PDEs [70,72] and the steady Navier-Stokes equations [71]. A numerical example from [70] is given in Figure 10.

Superconvergence based a posterior error estimator was further developed in [67] for CVT/CVDT based meshes, utilizing their superconvergence properties as shown in [66]. This leads to another CVT/CVDT based adaptive finite element algorithm for numerical PDEs. For a number of model second-order elliptic problems with complex geometries and various singular solutions, the convergence of the adaptive algorithm was demonstrated in [67].

For convection-dominated problems, one often encounters the difficulty that the overall accuracy of the numerical approximation is deteriorated by local singularities such as interior and boundary layers or sharp shock-like fronts. It is thus interesting to connect the density function to a posteriori error estimates and anisotropic metrics for determining 

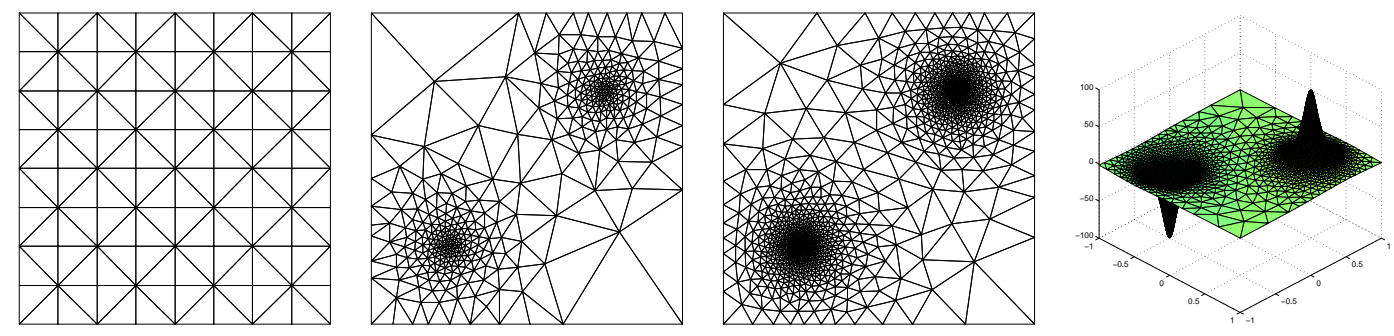

Figure 10: [70] Adaptively refined CfCVDT meshes (left three) and the approximate solution (right) for the equation $-\triangle u=f$ with an exact solution $u(x, y)=\frac{1.0}{(x-0.5)^{2}+(y-0.5)^{2}+0.01}+\frac{1.0}{(x+0.5)^{2}+(y+0.5)^{2}+0.01}$.

point distributions with special properties such as high aspect ratio spacings that would be useful for resolving boundary layers. Such an adaptive anisotropic meshing scheme for solving two-dimensional steady convection-dominated problems was developed in [94], in particular, the meshes are generated by the ACVDT algorithm [46] in combination with metric tensor information at each level of refinement. Some preliminary results have shown that the adaptive ASCVT approach results in substantial improvements compared to using regular isotropic CVT meshes; see Figure 11 for an illustration. The metric tensors are computed from the approximate solutions at each level using a formula suggested in $[62]$.
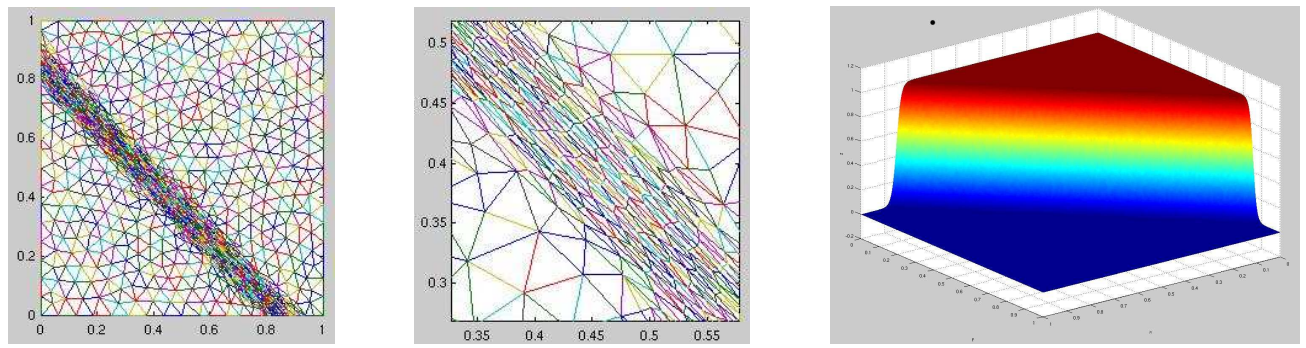

Figure 11: [94] An ACVDT mesh (left), the zoomed one (middle) and the approximate solution (right) generated by the ACVDT-based adaptive algorithm for a convection-dominated problem that has an interior layer.

\subsection{CVT-based model reduction}

Solutions of nonlinear complex systems such as those governed by systems of nonlinear partial differential equations are expensive with respect to both storage and CPU costs. As a result, it is difficult if not impossible to deal with a number of situations such as continuation or homotopy methods for computing state solutions, parametric studies of state solutions, optimization and control problems multiple state solutions), and real-time feedback control settings. Not surprisingly, a lot of attention has been paid to reducing the costs of the nonlinear state solutions by using reduced-order models for the state, i.e., low-dimensional approximations to the state. 
The type of reduced-order modeling we have in mind proceeds along the following lines. One first chooses a reduced basis $\left\{u_{i}\right\}_{i=1}^{K} ; K$ is hopefully very small compared to the usual number of degrees of freedom encountered in a finite element method. Next, one seeks an approximation $\widetilde{u}$ to the state of the form $\widetilde{u}=\sum_{i=1}^{K} c_{i} u_{i} \in V \equiv \operatorname{span}\left\{u_{1}, \ldots, u_{K}\right\}$. Then, one determines the coefficients $\left\{c_{i}\right\}_{i=1}^{K}$ by solving the state equations in the set $V$, e.g., one could find a Galerkin solution of the state equations in a standard way, using $V$ for the space of approximations; the cost of such a computation would be very small if $d$ is small (ignoring the cost of the off-line determination of the reduced basis $\left\{u_{i}\right\}_{i=1}^{K}$.)

The remaining question is how does one determine the reduced basis? Typically one begins by generating a set of snapshots. The state of a complex system is determined by parameters that appear in the specification of a mathematical model for the system. Snapshot sets consist of (expensive computational or, in principle, even experimental) state solutions corresponding to several parameter values and/or evaluated at several values time instants. Thus, typically, snapshots are very high-dimensional vectors of coefficients or nodal values that determine the approximate solution of the PDE obtained by a finite element or other method.

In some approaches, the snapshot set itself forms the reduced basis $[87,95,101]$. However, in some instances, snapshot sets contain lots of redundant information which we would like to remove. In this sense, we are faced with a data compression problem, namely, how do we extract a very low-dimensional basis set that captures the information contained in a snapshot set that may have cardinality in the hundreds or even thousands? One popular approach for this purpose is the proper orthogonal decomposition method [14, 75, 109] which is a projection method closely related to the singular value decomposition.

Alternately, one can apply the CVT concept which is a clustering approach to data compression. From a given snapshot set $\left\{U_{j}\right\}_{j=1}^{J}$ of vectors in $\mathbb{R}^{N}, N \gg 1$, a smaller set of vectors $\left\{u_{i}\right\}_{i=1}^{K}, K \ll J$, also belonging to $\mathbb{R}^{N}$, that are the generators of a CVT. The set $\left\{u_{i}\right\}_{i=1}^{K}$ is then the basis used for reduced order modeling. This basis is optimal in the sense that it minimizes the clustering energy for the given set of snapshots. In Figure 12 we reproduce, for two typical flow examples, plots given in [11] for the error vs. time for different choices for the number of CVT reduced basis vectors $K$.
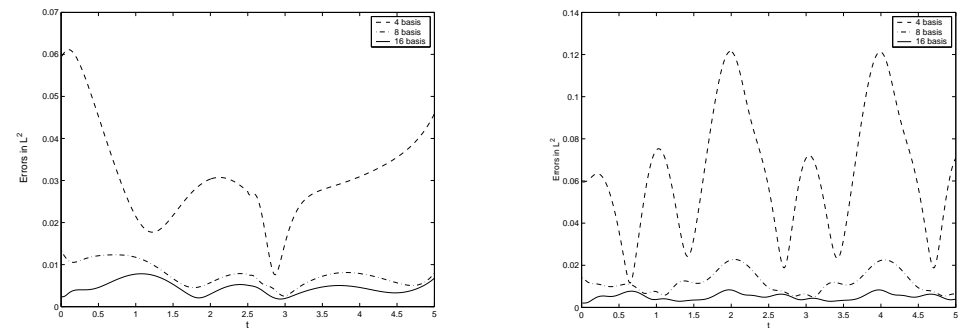

Figure 12: [11] For two typical Navier-Stokes problems, the $L^{2}$ difference between a CVT reduced basis solution (based on 500 snapshots) and the solution obtained using a finite element method (using about 5,000 degrees of freedom) vs. time and the number of reduced basis vectors. 
We have already mentioned in Sections 2.3 and 2.2 that the concept of centroidal Voronoi tessellations can be extended to more general notions of Voronoi generators and distance. This allows us to combine POD and CVT with the goal of taking advantage of the best features of both approaches. In $[33,34]$, the CVT concept was combined with the POD method into the hybrid CVOD method for model reduction. Briefly, the Voronoi generators and therefore centroids are defined by the POD basis, i.e., they are the space spanned by those vectors, within the Voronoi tessellations which contain subspaces formed from snapshots. The CVOD concept has been further studied in $[11,12,77,103]$ for a variety of physical models including the nonlinear Burger's equation and the Navier-Stokes equations. However, there remains much to be done to realize the full potential of the CVOD method.

\subsection{CVT-based image segmentation and analysis}

The classical image segmentation methods can be categorized into two major classes: edge-based methods such as edge detection and thresholding, and region-based models such as region growth and level set based active contour methods [23]. CVT-based image segmentation methods fall into the latter category. In its simplest form, the basic CVT model reduces to the well-known $k$-means clustering technique. However, by viewing the latter within the CVT context, very useful generalizations and improvements can be easily made. Some CVT-based algorithms were discussed in [38] for image compression and segmentation and multichannel image restoration, including the incorporation of cluster-dependent weights, the incorporation of averaging techniques to treat noisy images, and extensions to treat multichannel data. A CVT-based technique, called VOronoi Image SEgmentation (VOISE) [57], was successfully applied to the automatic detection and segmentation of Jupiter's auroral emissions. The algorithm consists of an iterative procedure that dynamically constructs a tessellation of the image plane based on a Voronoi diagram, until the intensity of the underlying image within each region is classified as homogeneous. The computed tessellations allow the extraction of quantitative information about the auroral features. Other application of CVT-based image analysis methods include adaptive spatial binning of integral-field spectroscopic data [20] and of X-Ray data [26].

More recently, an edge-weighted CVT (EWCVT) model for image segmentation was proposed in [121]; in particular, this new model combines the image intensity information together with the length of cluster boundaries and can handle very sophisticated situa-

tions. In the EWCVT model, the objective energy - the edge-weighted clustering energy $\widehat{E}$, consists of two parts, the classical clustering energy $E_{c}$ similar to (1.3) (but in the discrete sense) in color space and the weighted total length of the boundaries $E_{b}$ between clusters, i.e., $\widehat{E}=E_{c}+E_{b}$. The second component is also called the edge energy which is defined to be the weighted summation over every pixel $p$ of the image of the number of pixels in a certain neighborhood $\mathbb{N}(p, r)$ of $p$ but belonging to a different cluster. Extensive image segmentation experiments were presented in [121] to demonstrate the efficiency, effectiveness, robustness, and flexibility of the EWCVT-based method. An example result is provided in Figure 13. 

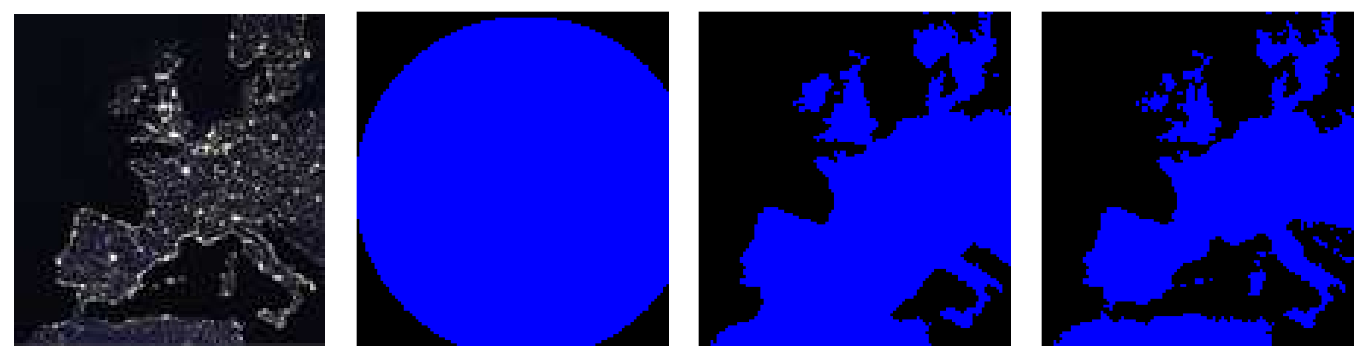

Figure 13: [121] Detection of land from the "Europe-at-night" image using the EWCVT model. From left to right: the original image, the initial segmentation, an intermediate segmentation, and the final segmentation.

\section{Concluding Remarks}

Although tremendous progress, as briefly summarized in this paper, has been made in the last decade on the generalization, construction, and analysis of CVTs, there are still many interesting questions waiting to be answered. Naturally, there is also no doubt that, in the future, there will be many more fields of application in which CVTs will be play in prominent role.

\section{Acknowledgments}

This work was supported by the US Department of Energy Office of Science Climate Change Prediction Program through grant numbers DE-FG02-07ER64431 and DE-FG0207ER64432, and the US National Science Foundation under grant numbers DMS-0609575 and DMS-0913491.

\section{References}

[1] P. Alliez, D. Cohen-Steiner, M. Yvinec, And M. Desbrun, Variational tetrahedral meshing, in Proceedings of SIGGRAPH, 2005, pp. 617-625.

[2] L. Antani, C. Delage, And P. Alliez, Mesh sizing with additively weighted Voronoi diagrams, in Proceedings of Meshing Roundtable Conference, 2007, pp. 335-346.

[3] S. Arya And D. Mount, Approximate nearest neighbor searching, Proceedings of the Fourth Ann. ACM-SIAM Symposium on Discrete Algorithms (SODA), 1993, pp. 271-280.

[4] M. Ainsworth And J. Oden, A Posteriori Error estimation in Finite Element Analysis, Wiley \& Sons, 2002.

[5] P. Alliez, T. De Verdiere, O. Devillers, and M. Isengurg, Centroidal Voronol diagrams for isotropic surface remeshing, Graphic Models, 67 (2005), pp. 204-231.

[6] E. ABAYA AND G. Wise, Convergence of vector quantizers with application to optimal quantization, SIAM J. Appl. Math., 44 (1984), pp.183-189.

[7] T. BAKER, Automatic mesh generation for complex three-dimensional regions using a constrained Delaunay triangulation, Engrg. Comput., 5 (1989), pp. 161-175.

[8] G. Barlow, Hexagonal territories, Animal Behav., 22 (1974), pp. 876-878. 
[9] P. BAJCsy and N. AhuJA, Location- and density-based hierarchical clustering using similarity analysis, IEEE Trans. Pattern Anal. Mach. Intel., 20 (1980), pp. 1011-1015.

[10] F. Bullo AND J. CoRTEs, Adaptive and distributed coordination algorithms for mobile sensing networks, Lecture Notes in Control and Information Sciences 309 (2005) pp. 43-62.

[11] J. Burkardt, M. Gunzburger, And H.-C. LeE, Centroidal Voronoi tessellation-based reducedorder modeling of complex systems, SIAM J. Sci. Comput., 28 (2006), pp. 459-484.

[12] J. Burkardt, M. Gunzburger, AND H.-C. LEe, POD and CVT-based reduced-order modeling of Navier-Stokes flows, Comp. Methods Appl. Mech. Engrg., 196 (2006), pp. 337-355.

[13] J. Burkardt, M. Gunzburger, J. Peterson, and R. Brannon, Centroidal Voronoi point placement and associated zeroth, first, and second moment determination, Sandia National Laboratories Report, SAND2002-0099, 2002.

[14] G. Berkooz, P. Holmes, AND J. Lumley, The proper orthogonal decomposition in the analysis of turbulent flows, Ann. Rev. Fluid. Mech., 25 (1993), pp. 539-575.

[15] T. Belytschko, Y. Krongauz, D. Organ, M. Fleming, and P. Krysl, Meshless methods: an overview and recent developments, Comput. Methods Appl. Mech. Engrg., 139 (1996), pp. 3-47.

[16] H. Borouchaki And S. Lo, Fast Delaunay triangulation in three dimensions, Comp. Methods Appl. Mech. Engrg., 128 (1995), pp. 153-167.

[17] I. Boier-Martin, H. Rushmeier, and J. Jin, Parameterization of triangle Meshes over quadrilateral domains, Proceedings of the Eurographics/ACM SIGGRAPH Symp., 2004, pp. 193-203.

[18] E. BARNeS AND N. SLOANE, The optimal lattice quantizer in three dimensions, SIAM J. Algebraic Discrete Methods, 4 (1983), pp. 31-40.

[19] D. Cohen-Steiner, P. Alliez, And M. Desbrun, Variational shape approximation, ACM Transactions on graphics, 23 (2004), pp. 905-914.

[20] M. Cappellari and Y. Copin, Adaptive spatial binning of integral-field spectroscopic data using Voronoi tessellations, Monthly Notices of the Royal Astronomical Society, 342 (2003), pp. 345-354.

[21] Y. Choi And S. Kim, Node generation scheme for meshfree method by Voronoi diagram and weighted bubble packing, Proceedings of the Fifth U.S. National Congress on Computational Mechanics, Boulder, 1999.

[22] J. Cortes, S. Martinez, T. Karatas, and F. Bullo, Coverage control for mobile sensing networks, IEEE Trans. Robotics and Automation, 20 (2004), pp. 243-255.

[23] T. Chan, J. Shen, ANd L. Vese, Variational PDE models in image processing, Notices of AMS, American Mathematical Society, 50 (2003), pp. 14-26.

[24] Y. Chen, Z. WANG, AND J. Liang, Optimal dynamic actuator location in distributed feedback control of a diffusion process, Inter. J. Sensor Networks, 2 (2007), pp. 169-178.

[25] L. Chen and J. Xu, Optimal Delaunay triangulation, J. Comp. Math., 22 (2004), pp. 299-308.

[26] S. Diehl And T. StatLer, Adaptive binning of X-Ray data with weighted Voronoi tesselations, MNRAS, 368 (2006), pp. 497-510.

[27] Q. Du And M. Emelianenko, Acceleration schemes for computing the centroidal Voronoi tessellations, Numer. Linear Alg. Appl., 13 (2006), pp. 173-192.

[28] Q. Du AND M. Emelianenko, Uniform convergence of a nonlinear energy-based multilevel quantization scheme via centroidal Voronoi tessellations, SIAM J. Numer. Anal., 46 (2008), pp. 1483-1502.

[29] Q. Du, M. Emelianenko And L. Ju, Convergence of the Lloyd algorithm for computing centroidal Voronoi tessellations, SIAM J. Numer. Anal., 44 (2006), pp. 102-119.

[30] Q. Du, M. Emelianenko, H. Lee And X. WAnG, Ideal point distributions, best mode selections and optimal spatial partitions via centroidal Voronoi tessellations, Proceedings of the Second 
Inter. Symp. Voronoi Diagrams Sci. Engrg., Seoul, Korea, 2005, pp. 325-333.

[31] Q. Du, V. Faber, and M. Gunzburger, Centroidal Voronoi tessellations: Applications and algorithms, SIAM Review, 41 (1999), pp. 637-676.

[32] Q. Du And M. Gunzburger, Grid generation and optimization based on centroidal Voronoi tessellations, Applied Math. Comptut., 133 (2002), pp. 591-607.

[33] Q. Du And M. Gunzburger, Model reduction by proper orthogonal decomposition coupled with centroidal Voronoi tessellation, Proceedings of the Fluids Engineering Division Summer Meeting, FEDSM2002-31051, ASME, 2002.

[34] Q. Du And M. Gunzburger, Centroidal Voronoi tessellation based proper orthogonal decomposition analysis, Control and Estimation of Distributed Parameter Systems, Birkhauser, Basel, 2003, pp. 137-150.

[35] Q. Du, M. Gunzburger, And L. Ju, Meshfree, probabilistic determination of points, sets and support regions for meshless computing, Comput. Methods Appl. Mech. Engrg., 191 (2002), pp. 1349-1366.

[36] Q. Du, M. Gunzburger, And L. Ju, Constrained centroidal Voronoi tessellations on general surfaces, SIAM J. Sci. Comput., 24 (2003), pp. 1488-1506.

[37] Q. Du, M. Gunzburger, And L. Ju, Voronoi-based finite volume methods, optimal Voronoi meshes and PDEs on the sphere, Comput. Methods Appl. Mech. Engrg., 192 (2003), pp. 39333957.

[38] Q. Du, M. Gunzburger, L. Ju, AND X.-Q. WAng, Centroidal Voronoi tessellation algorithms for image compression, segmentation and multichannel restoration, J. Math. Imaging Vision, 24 (2006), pp. 177-194.

[39] O. Deussen, S. Hiller, C. van Overveld, and T. Strohthotte, Floating points: A method for computing stipple drawings, Comput. Graph. Forum, 19 (2000), pp. 41-50.

[40] Q. Du, Z.-H. HuANG, AND D. WANG, Mesh and solver coadaptation in finite element methods for anisotropic problems, Numer. Meth. for PDEs, 21 (2005), pp. 859-874.

[41] Q. Du AND L. Ju, Numerical simulations of the quantized vortices in a thin superconducting hollow sphere, J. Comput. Phys., 201 (2004), pp. 511-530.

[42] Q. Du AND L. Ju, Approximations of a Ginzburg-Landau model for superconducting hollow spheres based on spherical centroidal Voronoi tessellations, Math. Comp., 74 (2005), pp. 1257-1280.

[43] Q. Du AND L. Ju, Finite volume methods on spheres and spherical centroidal Voronoi meshes, SIAM J. Numer. Anal., 43 (2005), pp. 1673-1692.

[44] Q. Du AND D. WANG, Tetrahedral mesh generation and optimization based on centroidal Voronoi tessellations, Int. J. Numer. Methods Engrg., 56 (2003), pp. 1355-1373.

[45] Q. Du AND D. WANG, Constrained boundary recovery for 3D Delaunay triangulations, Int. J. Num. Method. In. Eng., 61 (2004), pp. 1471-1500.

[46] Q. Du AND D. WANG, Anisotropic centroidal Voronoi tessellations and their applications, SIAM. J. Sci. Comput., 26 (2005), pp. 737-761.

[47] Q. Du AND D. WANG, On the optimal centroidal Voronoi tessellations and the Gersho's conjecture in the three dimensional space, Comput. Math. Appl., 49 (2005), pp. 1355-1373.

[48] Q. Du AND D. WANG, Mesh optimization based on centroidal Voronoi tessellations, Inter. J. Numer. Anal. Model., 2 (2005), pp. 100-114.

[49] Q. Du AND D. WANG, Recent progress in robust and quality Delaunay mesh generation, $J$. Comput. Appl. Math., 195 (2006), pp.8-23.

[50] Q. Du AND X. WANG, Tessellation and clustering by mixture models and their parallel implementations, Proceedings of the Fourth SIAM Inter. Conf. Data Mining, Orlando, FL, 2004, SIAM, 2004, pp. 257-268. 
[51] Q. Du And X. WANG, Centroidal Voronoi tessellation based algorithms for vector fields visualization and segmentation, Proceedings of the IEEE Visualization, Austin, Texas, 2004, pp. 43-50.

[52] Q. Du And T. Wong, Numerical studies of the k-means algorithm, Comput. Math. Appl., 44 (2002), pp. 511-523.

[53] M. Emelianenko, L. Ju AND A. Rand, Nondegeneracy and weak global convergence of the Lloyd algorithm in $\mathbb{R}^{d}$, SIAM J. Numer. Anal., 46 (2008), pp. 1423-1441.

[54] P. FLEISCHER, Sufficient conditions for achieving minimum distortion in a quantizer, IEEE Int. Convention Record I (1964), pp. 104-111.

[55] A. Gersho, Asymptotically optimal block quantization, IEEE Trans. Inform. Theory, 25 (1979), pp. 373-380.

[56] P. Gruber, Optimum quantization and its applications, Adv. Math., 186 (2004), pp. 456-497.

[57] P. Guio And N. Achilleos, The VOISE algorithm: a versatile tool for automatic segmentation of astronomical images, Mon. Not. R. Astron. Soc., 2009, doi:10.1111/j.13652966.2009.15218.x.

[58] A. Gersho and R. Gray, Vector Quantization and Signal Compression, Kluwer, Boston, 1992.

[59] R. Gray, J. KiefFer, AND Y. Linde, Locally optimal block quantizer design, Inform. Control, 45 (1980), pp. 178-198.

[60] R. Gray and D. Neuhoff, Quantization, IEEE Trans. Inform. Theory, 44 (1998), pp. 23252383.

[61] A. HAusner, Simulating decorative mosaics, Proceedings of SIGGRAPH, 2001, pp. 573-578.

[62] W. Huang, Metric tensors for anisotropic mesh generation, J. Comput. Phys., 204 (2005), pp. 633-665.

[63] H. Hellwig, R. Engelmann, and O. Deussen, Contact pressure models for spiral phyllotaxis and their computer simulation, J. Theo. Biol., 240 (2005), pp. 489-500.

[64] H. Hellwig, M. Jeschke, And O. Deussen, A contact pressure model based on centroidal Voronoi diagrams for surfaces of revolution, Proceedings of the Fifth Annual Inter. Symp. Voronoi Diagrams Sci. Engr., 2008.

[65] S. Hiller, H. Hellwig, and O. Deussen, Beyond stippling - methods for distributing objects on the plane, Computer Graphics Forum, 22 (2003), pp. 515-522.

[66] Y. Huang, H. Qin, AND D. WANG, Centroidal Voronoi tessellation-based finite element superconvergence, Inter. J. Numer. Meths. Engrg., 76 (2008), pp. 1819-1839.

[67] Y. HuANG, H. Qin, D. WANG, AND Q. Du, Convergent adaptive finite element method based on centroidal Voronoi tessellations and superconvergence, preprint, 2009.

[68] L. Ju, Conforming centroidal Voronoi Delaunay triangulation for quality mesh generation, Inter. J. Numer. Anal. Model., 4 (2007), pp. 531-547.

[69] L. Ju, Q. Du, AND M. Gunzburger, Probabilistic methods for centroidal Voronoi tessellations and their parallel implementations, Parallel Comput., 28 (2002), pp. 1477-1500.

[70] L. Ju, M. Gunzburger, And W.-D. ZhaO, Adaptive finite element methods for elliptic PDEs based on conforming centroidal Voronoi Delaunay triangulations, SIAM J. Sci. Comput., 28 (2006), pp. 2023-2053.

[71] L. Ju, H.-C. LeE, AND L. Tian, Numerical simulations of the steady Navier-Stokes equations using adaptive meshing schemes, Int. J. Numer. Meth. Fluids, 56 (2008), pp. 703-721.

[72] L. Ju, W. Wu, AND W.-D. ZHAO, Adaptive finite volume methods for steady convection-diffusion equations with mesh optimization, Dis. Cont. Dyn. Sys. B, 11 (2009), pp. 669-690.

[73] J. KIEFFER, Uniqueness of locally optimal quantizer for log-concave density and convex error function, IEEE Trans. Infor. Theory, 29 (1983), pp. $42-47$.

[74] T. Kanungo, D. Mount, N. Netanyahu, C. Piatko, R. Silverman, and A. Wu, An efficient k- 
means clustering algorithm: Analysis and implementation, IEEE Trans. Pattern Anal. Mach. Intel., 24 (2002), pp. 881-892.

[75] K. Kunisch And S. Volkwein, Galerkin proper orthogonal decomposition methods for parabolic problems, Numer. Math., 90 (2001), pp. 117-148.

[76] F. Labelle AND J. Shewchuk, Anisotropic voronoi diagrams and guaranteed-quality anisotropic mesh generation. In Proc. 19th Symposium on Computational Geometry (SoCG), pages 191-200, New York, NY, USA, 2003. ACM Press.

[77] H.-C. LEE, S.-W. LeE AND G.-R. PIAO, Reduced-order modeling of Burger equations based on centroidal Voronoi Tessellation Int. J. Numer. Anal. Model., 4 (2007), pp. 559-583.

[78] D. Liberzon, Hybrid feedback stabilization of systems with quantized signals, Automatica, 39 (2003), pp. 1543-1554.

[79] W. LipsComb AND T. RingleR, An incremental remapping transport scheme on a spherical geodesic grid, Monthly Weather Reiview, 133 (2005), pp. 2335-2350.

[80] D. Liu AND J. Nocedal, On the limited memory BFGS method for large scale optimization, Mathematical Programming: $A$ and B, 45 (1989), pp. 503-528.

[81] Y. Liu, W. WANG, B. LevY, F. SUnG, AND D-M. YAN, On centroidal Voronoi tessellation - Energy smoothness and fast computation, ACM Trans. Graphics, to appear.

[82] S. LLoyd, Least squares quantization in PCM, IEEE Trans. Inform. Theory, 28 (1982), pp. 129-137.

[83] R. LOHNer and P. PARIKh, Generation of three-dimensional unstructured grids by the advancing-front method, Int. J. Num. Meth. Fluid, 8 (1988), pp. 1135-1149.

[84] L. Lu, B. Levy AND W.-B. WANG, Centroidal Voronoi tesselation of line segments and graphs, INRIA-ALICE Technical report, 2009.

[85] D. Luenberger, Linear and Nonlinear Programming, Addison-Wesley, Reading, MA, 1984.

[86] J. MacQueEn, Some methods for classification and analysis of multivariate observations, Proceedings of the Fifth Berkeley Symp. Math. Stat. and Prob., Vol I, Ed. by L. Le Cam and J. Neyman, University of California, 1967, pp. 281-297.

[87] Y. Maday, A. Patera, AND G. Turinici, A priori convergence theory for reduced-basis approximations of single-parameter elliptic partial differential equations, J. Sci. Comput., 17 (2002) pp. 437-446.

[88] M. Murphy, D. Mount, And C. Gable, A point-placement strategy for Conforming Delaunay tetrahedralization, Inter. J. Comput. Geom. Appl., 11 (2001), pp. 669-682.

[89] P. Morin, R. Nochetto, AND K. Siebert, Data oscillation and convergence of adaptive finite element methods, SIAM J. Numer. Anal., 38 (2000), pp. 466-488.

[90] M. Moriguchi and K. Sugihara, Constructing centroidal Voronoi tessellations on surface meshes, Studies in Computational Intelligence, 158 (2009), pp. 235-245.

[91] D. Newman, The hexagon theorem, IEEE Trans. Inform. Theory, 28 (1982), pp. 137-139.

[92] F. Nielson, J. Boissonnat and R. Nock, Bregman Voronoi Diagrams: Properties, Algorithms and Application, preprint, 2007.

[93] H. Nguyen, J. Burkardt, M. Gunzburger, L. Ju, and Y. Saka, Constrained CVT meshes and a comparison of triangular mesh generators, Comput. Geom.: Theory Appl., 42 (2009), pp. $1-19$.

[94] H. Nguyen, M. Gunzburger, L. Ju, And J. Burkardt, Adaptive anisotropic meshing for steady convection-dominated problems, Comput. Methods Appl. Mech. Engrg., 198 (2009), pp. 29642981.

[95] A. Noor, Recent advances in reduction methods for nonlinear problems, Comput. \& Struc., 13 (1981), pp. 31-44.

[96] А. Окаве, B. Воots, K. Sugihara, and S. Chiu, Spatial Tessellations: Concepts and Applications 
of Voronoi Diagrams, 2nd edition, Wiley, Chichester, 2000.

[97] V. Ostromoukhov, C. Donohue, And P. Jodoin, Fast hierarchical importance sampling with blue noise properties, ACM Trans. Graphics, 23 (2004), pp. 488-495.

[98] Y. OUYANG, Design of vehicle routing zones for large-scale distribution systems, Transportation Research B: Methodological, 41 (2007), pp. 1079-1093.

[99] Y. OuYAng And C. Daganzo, Discretization and validation of the continuum approximation scheme for terminal system design, Transportation Science, 40 (2006), pp. 89-98.

[100] S. Osher And N. Paragios, Geometric Level Set Methods in Imaging, Vision and Graphics, Springer, 2002.

[101] J. Peterson, The reduced basis method for incompressible flow calculations, SIAM J. Sci. Stat. Comput., 10 (1989), pp. 777-786.

[102] G. Peyre And L. Cohen, Surface segmentation using geodesic centroidal tesselation, Proceedings of the Second Inter. Symp. 3D Data Processing, Visualization and Transmission, 2004, pp. 995-1002.

[103] G. PIAO, H.-C. LEE AND Q. Du, Adaptive CVT-based reduced-order modeling of Burgers equation, J. Korean SIAM, 13 (2009), pp.141-159.

[104] P.-O. Persson And G. Strang, A simple mesh generator in Matlab, SIAM Review, 46 (2004), pp. 329-345.

[105] R. RENKA, ALGORITHM 772. STRIPACK: Delaunay triangulation and Voronoi diagrams on the surface of a sphere, ACM Trans. Math. Soft., 23 (1997), pp. 416-434.

[106] T. RingLER, Comparing truncation error to PDE solution error on spherical Voronoi Tessellations, Tech report, Dept. Atmospheric Sci., Colorado State Univ., 2003.

[107] V. Romero, J. Bourkardt, M. Gunzburger and J. Peterson, Comparison of pure and "Latinized" centroidal Voronoi tessellation against various other statistical sampling methods, Reliab. Engrg. Sys. Safe., 91 (2006), pp. 1266-1280.

[108] T. Ringler, L. Ju, And M. Gunzburger, A multi-resolution method for climate system modeling, Ocean Dynamics, 58 (2008), pp. 475-498.

[109] M. Rathinam and L. Petzold, A new look at proper orthogonal decomposition, SIAM J. Numer. Anal., 41 (2003), pp. 1893-1925.

[110] A. SECORD, Weighted Voronoi stippling, Proceedings of the Second International Symposium on Non-Photorealistic Animation and Rendering, NPAR, 2002.

[111] J. Shewchuк, Triangle: Engeering a 2D quality mesh generator and Delaunay triangulator, Lecture Notes in Comput. Sci., 1148 (1996), Springer-Verlag, New York, pp. 203-222.

[112] M. Samozino, M. Alexa, P. Alliez, and M. Yvinec, Reconstruction with Voronoi centered radial basis functions, Proceedings of Sympos. Geom. Processing, 2006, pp. 51-60.

[113] M. Shephard And M. Georges, Automatic three-dimensional mesh generation technique by the finite element octree technique, Inter. J. Numer. Meths. Engrg., 32 (1991), pp. 709-749.

[114] J. SABIN AND R. GRAY, Global convergence and empirical consistency of the generalized Lloyd algorithm, IEEE Trans. Inform. Theory, 32 (1986) pp. 148-155.

[115] Y. SAKA, M. GunZBurger, AND J. BuRKARDT, Latinized, improved LHS, and CVT point sets in hypercubes, Int. J. Numer. Anal. Model, 4 (2007), pp. 729-743.

[116] S. VAlette AND J. Chassery, Approximated centroidal Voronoi diagrams for uniform polygonal mesh coarsening, Computer Graphics forum, 23 (2004), pp. 381-389.

[117] S. Valette, J. Chassery, And R. Prost, Generic remeshing of 3D triangular meshes with metric-Dependent discrete Voronoi diagrams, IEEE Trans. Visual. Comput. Graphics, 14 (2008), pp. 369-381.

[118] D. WANG, A new method for efficient generation of high quality surface triangular meshes, Comms. Comput. Phys., 1 (2006), pp. 716-735. 
[119] X. Wu, On Convergence of Lloyd's Method I, IEEE Trans. Inform. Theory, 38 (1992), pp. 171-174.

[120] D. Wang, O. Hassan, K. Morgan, and N. Weatherill, EQSM: An efficient high quality surface grid generation method based on remeshing, Comput. Methods Appl. Mech. Engrg., 195 (2006), pp. 5621-5633.

[121] J. WANG, L. Ju, AND X. WANG, An edge-weighted centroidal Voronoi tessellation model for image segmentation, IEEE Trans. Image Processing, 18 (2009), pp. 1844-1858.

[122] D. Williamson, J. Drake, J. Hack, R. Jakob, and P. Swarztrauber, A standard test set for numerical approximations to the shallow water equations in spherical geometry, $J$. Comput. Phys., 102 (2001), pp. 211-224. 